\title{
TWO LOWER BOUNDS ABOUT SINGULAR SUBSPACES
}

\section{Chunguang Ren}

Abstract. Cai and Zhang establish two lower bounds for $\sin \Theta$ distances with spectral and Frobenius norms (Cai, T. T. and Zhang, A., Rate-optimal perturbation bounds for singular subspaces with applications to high-dimensional statistics, The Annals of Statistics, Vol. 46, No. 1 (2018) $60-89)$. We provide two lower bounds under any unitarily invariant norm. It turns out that our estimation is better in some sense.

Mathematics subject classification (2010): 15A18, 15A42, 34D15.

Keywords and phrases: Lower bounds, unitarily invariant norm, $\sin \Theta$ distance, singular value decomposition.

\section{REFERENCES}

[1] T. T. CAI AND A. R. ZHANG, Rate-optimal perturbation bounds for singular subspaces with applications to high-dimensional statistics, The Annals of Statistics. 46 (2018), 60-89.

[2] T. T. CAI AND A. R. Zhang, Supplement to Rate-optimal perturbation bounds for singular subspaces with applications to high-dimensional statistics, 2018. doi:10.1214/17-AOS1541SUPP.

[3] R. A. Horn And C. R. Johnson, Topics in Matrix Analysis, Cambridge University Press, Cambridge, 1991.

[4] R. A. Horn And C. R. Johnson, Matrix analysis (Second Edition), Cambridge University Press, Cambridge, 2012.

[5] Y. M. LiU AND C. G. Ren, An optimal perturbation bound, Mathematical Methods in the Applied Sciences 42 (2019), 3791-3798. 